\title{
CORRESPONDENCE The role of CD6 in autoimmune diseases
}

\author{
Cellular \& Molecular Immunology (2018) 15:1001-1002; \\ https://doi.org/10.1038/s41423-018-0015-1
}

In a recent publication in the Cellular and Molecular Immunology, Consuega-Fernandez and colleagues present new data from patients with psoriasis, linking the CD6 lymphocyte surface structure to the pathogenesis of this disease. ${ }^{1}$ In this commentary, I will discuss these new findings in the context of accumulating evidence for important roles of CD6 in a variety of autoimmune disorders. Although CD6 was one of the earliest "CD antigens" to be described, it is only recently that attention has refocused on the potential for CD6 as a treatment target in immune-mediated diseases.

\section{GENETIC ASPECTS OF CD6}

CD6 is located on chromosome 11 adjacent to CD5, which encodes a homologous lymphocyte surface glycoprotein. Both CD6 and CD5 are expressed by most mature T cells and a B cell subset. The molecular mass of CD6 is $105-130 \mathrm{kDa}$, which reflects variable glycosylation and the existence of multiple isoforms generated by alternative splicing of the CD6 transcript. The extracellular portion of CD6 includes 3 scavenger receptor domains, whereas the intracellular portion, although devoid of kinase activity, has sites for the phosphorylation and recruitment of signal transduction proteins.

In 2009, CD6 was identified as a susceptibility locus in multiple sclerosis ${ }^{2}$, and more recently, CD6 was identified as a susceptibility locus in Behcet's disease. ${ }^{3}$ Epigenetic regulation of CD6 expression is implied by its progressive upregulation during thymocyte maturation. Hypomethylation of CD6 in an autoimmune disease has been shown in the vasculitic lesions of giant cell arteritis, together with hypomethylation of various other genes related to $\mathrm{T}$ cell activation and effector $\mathrm{T}$ cell differentiation. ${ }^{4}$

In their recent paper, Consegua-Fernandez and colleagues now provide evidence that $C D 6$ is a severity gene in psoriasis. CD6associated single nucleotide polymorphisms controlled the extent and severity of skin involvement and the incidence of psoriatic arthritis in a cohort of psoriasis patients. ${ }^{1}$ It will now be important to undertake functional genomic studies to elucidate the mechanisms underlying these observations, which could include regulation of CD6 expression and perhaps differential utilization of alternative splicing to generate an altered balance of CD6 isoforms.

\section{ROLES OF CD6 IN LYMPHOCYTE ACTIVATION}

Although there is some evidence that CD6 can augment signal transduction through the T cell receptor complex ${ }^{5,6}$, recent studies in CD6-deficient mice indicate that CD6 attenuates the early stages of $T$ cell activation but also enhances Th1 and Th17 CD4 effector cell differentiation. ${ }^{7}$ The cytoplasmic region of CD6 can be phosphorylated by multiple kinases ${ }^{5,8}$, and many details of how CD6 participates in T cell signaling remain unclear, including the positioning of CD6 with respect to the "immunological synapse" formed through contact of the $\mathrm{T}$ cell with an antigen-presenting cell (APC). There is also evidence for a role of CD6 in thymic selection ${ }^{9,10}$ and in signaling events in CD6+ B cells. ${ }^{11}$

\section{CD6 LIGANDS-CD166 AND CD318}

CD166, also known as ALCAM (activated leucocyte cell adhesion molecule), was the first endogenous ligand of CD6 to be identified. $^{12}$ CD166, a member of the immunoglobulin gene superfamily, is widely expressed on cells of the immune system, including activated $\mathrm{T}$ and $\mathrm{B}$ lymphocytes, and on many types of mesenchymal, epithelial, and endothelial cells in various organs and tissues. Indications that a second ligand of CD6 might exist arose initially from functional studies in which $\mathrm{T}$ cell adhesion to keratinocytes or synovial fibroblasts was found to be dependent on CD6 but not on CD166. ${ }^{13,14}$ Moreover, these interactions involved a CD6-ligand whose expression was increased by cellular exposure to gamma-interferon, a cytokine that does not upregulate CD166. Subsequently, a monoclonal antibody was generated against gamma-interferon-treated synovial fibroblasts that identified a $130 \mathrm{kDa}$ molecule that was also bound to a CD6Ig fusion protein. ${ }^{15}$ This molecule was recently shown to be CD318, and CD318 has now been established as a second ligand of CD6 by multiple biochemical and molecular approaches. ${ }^{16}$ CD318 is expressed by a variety of tissue cell types with which $T$ cells interact in organ-targeted autoimmune diseases, but it is not expressed on cells of the immune system such as lymphocytes and myeloid cells. CD166 and CD318 bind to distinct domains of CD6, and it appears possible that CD6 could bind both ligands concurrently. ${ }^{16} \mathrm{CD} 6$ can also interact with innate immune stimuli, including both pathogen-associated (such as bacterial peptidoglycans) $)^{17}$ and damage-associated (such as galectins) ${ }^{18}$ stimuli. The functional significance of these binding properties in host defenses is not yet well understood.

IMPORTANCE OF CD6 IN MOUSE MODELS OF AUTOIMMUNITY Recent data show that CD6-/- mice are protected from the experimental allergic encephalomyelitis (EAE) model of multiple sclerosis (MS). The CD6-deficient mice have both a reduction in the Th1/Th17 response to myelin antigens and decreased infiltration of tissue-damaging immune cells in the central nervous system. ${ }^{7}$ The involvement of CD6 itself in EAE was further demonstrated in CD6-/- mice in which a human CD6 transgene was expressed in T cells. When EAE was induced in these mice, its progression was halted by the administration of a mouse antihuman CD6 monoclonal antibody that recognizes domain 1 of CD6-the domain to which CD318 but not CD166 binds. ${ }^{7}$ Moreover, CD318-/- mice were robustly protected from $\mathrm{EAE}^{16}$, but CD166-/- mice have been reported to have worse EAE than controls. ${ }^{19}$ Thus, the role of CD6 in fomenting organ-targeted autoimmunity may specifically reflect CD6 interaction with CD318, which is expressed on key tissue cells in these diseases-neurons and endothelial cells in the brain, synovial fibroblasts in the joint and keratinocytes in the skin ${ }^{13-16}$. Studies of a variety of other autoimmune models in CD6-/- mice are necessary. Collageninduced arthritis (CIA), a model of rheumatoid arthritis (RA), was reportedly worsened in $\mathrm{CD6}-/-$ mice $^{10}$, but that work was 
performed using the $\mathrm{C} 57 \mathrm{BI}$ strain in which an attenuated form of CIA is observed ${ }^{10}$; data from the DBA strain in which induction of $\mathrm{CIA}$ is robust and optimal will be of interest.

Consuega-Fernandez and colleagues also report observations in an imiquimod-induced mouse model of psoriasis, in which attenuated disease was observed in CD6-/- mice. As in the EAE studies mentioned above, reduction of the pathogenic Th1/Th17 response was observed. ${ }^{1}$

\section{CD6 BLOCKADE-DATA FROM CLINICAL TRIALS IN HUMANS}

In 1980s, an lgM anti-CD6 was tested in clinical trials on $\mathrm{MS}^{20}$ and allograft rejection ${ }^{21}$, but the small size and uncontrolled design of those studies precluded determination of therapeutic efficacy. More recently, an anti-CD6 monoclonal antibody termed itolizumab has been extensively studied in humans with psoriasis and was demonstrated to be of significant clinical benefit ${ }^{22}$, leading to approval for treatment of psoriasis in India. Anti-CD6 monoclonal antibodies do not significantly deplete $T$ cells in vivo ${ }^{7}$, likely due to capping and internalization of CD6/anti-CD6 before cytotoxic effector mechanisms can be mobilized, and this lack of cell depletion could lead to a safety advantage for CD6-directed monoclonals.

\section{FUTURE DIRECTIONS}

The new evidence for CD6 as a key molecule in the pathogenesis of some autoimmune diseases should prompt investigation of a wider range of human and rodent model T-cell-driven conditions to determine how extensive the importance of CD6 and its ligands might be across the broader spectrum of pathogenic autoimmunity. CD6, CD166, and CD318 all exist in soluble and transmembrane forms, and the soluble forms may also be important in disease. For example, sCD318 is elevated in synovial fluid from patients with RA and juvenile inflammatory arthritis (JIA) and is chemotactic for T cells at concentrations similar to the levels in the synovial fluid of inflamed joints. ${ }^{16}$

A novel ligand of CD166 (ILT3-immunoglobulin-like transcript 3) was recently described that, like CD6, is also expressed on $\mathrm{T}$ cells ${ }^{23}$; whether these two $\mathrm{T}$ cell receptors for CD166 function cooperatively or antagonistically is yet to be determined.

Importantly, the success of an anti-CD6 monoclonal antibody in the treatment of psoriasis seems likely to spur the testing of this and other CD6-targeting biologics in an expanding spectrum of human immune-mediated diseases.

David A. Fox ${ }^{1}$

${ }^{1}$ Division of Rheumatology and Clinical Autoimmunity Center of Excellence, University of Michigan, Ann Arbor, MI, USA Correspondence: David A. Fox (dfox@umich.edu)

\section{ADDITIONAL INFORMATION}

Competing interests: The author declares no conflict interest.

Publisher's note: Springer Nature remains neutral with regard to jurisdictional claims in published maps and institutional affiliations.

\section{REFERENCES}

1. Consuegra-Fernandez, M. et al. Genetic and experimental evidence for the involvement of the CD6 lymphocyte receptor in psoriasis. Cell. Mol. Immunol. 14, 1-9 (2017).

2. De Jager, P. L. et al. Meta-analysis of genome scans and replication identify CD6, IRF8 and TNFRSF1A as new multiple sclerosis susceptibility loci. Nat. Genet. 41, 776-782 (2009).

3. Zheng, M. et al. Genetic polymorphisms of cell adhesion molecules in Behcet's disease in a Chinese Han population. Sci. Rep. 6, 24974 (2016).

4. Coit, P., De Lott, L. B., Nan, B., Elner, V. M. \& Sawalha, A. H. DNA methylation analysis of the temporal artery microenvironment in giant cell arteritis. Ann. Rheum. Dis. 75, 1196-1202 (2016).

5. Osorio, L. M., Ordonez, C., Garcia, C. A., Jondal, M. \& Chow, S. C. Evidence for protein tyrosine kinase involvement in CD6-induced $\mathrm{T}$ cell proliferation. Cell. Immunol. 166, 44-52 (1995).

6. Ibanez, A. et al. Mitogen-activated protein kinase pathway activation by the CD6 lymphocyte surface receptor. J. Immunol. 177, 1152-1159 (2006).

7. Li, Y. et al. CD6 as a potential target for treating multiple sclerosis. Proc. Natl Acad. Sci. USA 114, 2687-2692 (2017).

8. Kobarg, J., Whitney, G. S., Palmer, D., Aruffo, A. \& Bowen, M. A. Analysis of the tyrosine phosphorylation and calcium fluxing of human CD6 isoforms with different cytoplasmatic domains. Eur. J. Immunol. 27, 2971-2980 (1997).

9. Singer, N. G. et al. CD6: expression during development, apoptosis and selection of human and mouse thymocytes. Int. Immunol. 14, 585-597 (2002).

10. Orta-Mascaro, M. et al. CD6 modulates thymocyte selection and peripheral T cell homeostasis. J. Exp. Med. 213, 1387-1397 (2016).

11. Osorio, L. M., De Santiago, A., Aguilar-Santelises, M., Mellstedt, H. \& Jondal, M. $\mathrm{CD} 6$ ligation modulates the $\mathrm{BCl}-2 / \mathrm{Bax}$ ratio and protects chronic lymphocytic leukemia B cells from apoptosis induced by anti-lgM. Blood 89, 2833-2841 (1997).

12. Bowen, M. A. et al. Cloning, mapping, and characterization of activated leukocytecell adhesion molecule (ALCAM), a CD6 ligand. J. Exp. Med. 181, 2213-2220 (1995).

13. Singer, N. G. et al. CD6 dependent interactions of T cells and keratinocytes: functional evidence for a second CD6 ligand on gamma-interferon activated keratinocytes. Immunol. Lett. 58, 9-14 (1997).

14. Joo, Y. S. et al. Evidence for the expression of a second CD6 ligand by synovial fibroblasts. Arthritis Rheum. 43, 329-335 (2000).

15. Saifullah, M. K. et al. Expression and characterization of a novel CD6 ligand in cells derived from joint and epithelial tissues. J. Immunol. 173, 6125-6133 (2004).

16. Enyindah-Asonye, G. et al. CD318 is a ligand for CD6. Proc. Natl Acad. Sci. USA 114, E6912-E6921 (2017).

17. Sarukhan, A. et al. Pattern recognition by CD6: a scavenger-like lymphocyte receptor. Curr. Drug Targets 17, 640-650 (2016).

18. Escoda-Ferran, C. et al. Modulation of CD6 function through interaction with Galectin-1 and -3. FEBS Lett. 588, 2805-2813 (2014).

19. Lecuyer, M. A. et al. Dual role of ALCAM in neuroinflammation and blood-brain barrier homeostasis. Proc. Natl Acad. Sci. USA 114, E524-E533 (2017).

20. Hafler, D. A. et al. Immunologic responses of progressive multiple sclerosis patients treated with an anti-T-cell monoclonal antibody, anti-T12. Neurology 36, 777-784 (1986).

21. Carpenter, C. B. et al. Monoclonal anti-T12 antibody as therapy for renal allograft rejection. Trans. Assoc. Am. Physicians 96, 84-92 (1983).

22. Krupashankar, D. S. et al. Efficacy and safety of itolizumab, a novel anti-CD6 monoclonal antibody, in patients with moderate to severe chronic plaque psoriasis: results of a double-blind, randomized, placebo-controlled, phase-III study. J. Am. Acad. Dermatol. 71, 484-492 (2014).

23. Xu, Z. et al. ILT3.Fc-CD166 interaction induces inactivation of p70 S6 kinase and inhibits tumor cell growth. J. Immunol. 200, 1207-1219 (2018). 